\title{
Consequências do uso contínuo de anticoncepcional: um alerta as mulheres
}

\author{
Consequences of continuous use of contraceptive: an alert to women
}

Consecuencias del uso continuo de anticonceptivos: una alerta para las mujeres

\author{
Adalberto Silva Barbosa \\ ORCID: https://orcid.org/0000-0003-4929-8226 \\ Faculdade Guaraí, Brasil \\ E-mail: asbeto.silva@gmail.com \\ Camila Teixeira De Oliveira Penna Chaves \\ ORCID: https://orcid.org/0000-0002-3008-2591 \\ Faculdade Guaraí, Brasil \\ E-mail: pennachavescamila@gmail.com
}

\begin{abstract}
Resumo
A contracepção hormonal é um dos mais utilizado pelas mulheres do Brasil e do mundo. No Brasil, são estimados $23 \%$ das mulheres com idade considerada reprodutiva (10 a 49 anos) usuárias dos anticoncepcionais hormonais. Os efeitos colaterais mais frequentes, a esses medicamentos são: Os efeitos colaterais mais frequentes, associados a esses medicamentos são: dores de cabeça, náuseas, vômitos, tonturas, irritabilidade, aumento do apetite com consequência ganho de peso, alterações no apetite sexual e queda considerável de cabelo. A falta de conhecimento sobre os efeitos colaterais oriundos do uso contínuo dos contraceptivos hormonais orais tem afetado sua eficácia, é essencial que essas mulheres sejam alertadas sobre o uso desse método contraceptivo, a forma mais correta de tomá-los, quem pode ou não fazer uso e especialmente, informar corretamente dos riscos e benefícios que os contraceptivos orais podem ter. Este trabalho teve como objetivo, evidenciar e relatar o conhecimento de acadêmicas da Faculdade de Guaraí - TO, sobre as consequências do uso prolongado e contínuo de anticoncepcional oral, que por muitas vezes é utilizado sem prescrição médica. Torna-se imprescindível o papel dos profissionais da saúde na hora de orientar e indicar o anticoncepcional ideal a cada mulher, afim de evitar a contracepção de forma errônea e gerando assim diversos efeitos e problemas a vida de cada mulher.
\end{abstract}

Palavras-chave: Conhecimento; Anticoncepcional oral; Uso prolongado; Consequências.

\begin{abstract}
Hormonal contraception is one of the most used by women in Brazil and the world. In Brazil, an estimated $23 \%$ of women with a reproductive age (10 to 49 years) are users of hormonal contraceptives. The most frequent side effects of these medications are: The most frequent side effects associated with these medications are: headache, nausea, vomiting, dizziness, irritability, increased appetite resulting in weight gain, changes in sexual appetite and fall handsome of hair. The lack of knowledge about the side effects arising from the continued use of oral hormonal contraceptives has affected its effectiveness, it is essential that these women are warned about the use of this contraceptive method, the most correct way to take them, who can or cannot use them and especially, correctly informing about the risks and benefits that oral contraceptives may have. This study aimed to demonstrate and report the knowledge of academics at the Faculty of Guaraí - TO, about the consequences of prolonged and continuous use of oral contraceptives, which are often used without a medical prescription. The role of health professionals is essential when it comes to guiding and indicating the ideal contraceptive for each woman, in order to avoid erroneous contraception and thus generating different effects and problems in each woman's life.
\end{abstract}

Keywords: Knowledge; Oral contraceptive; Extended use; Consequences.

\section{Resumen}

La anticoncepción hormonal es una de las más utilizadas por las mujeres en Brasil y el mundo. En Brasil, se estima que el $23 \%$ de las mujeres en edad reproductiva (10 a 49 años) son usuarias de anticonceptivos hormonales. Los efectos secundarios más frecuentes de estos medicamentos son: Los efectos secundarios más frecuentes asociados con estos medicamentos son: dolor de cabeza, náuseas, vómitos, mareos, irritabilidad, aumento del apetito que resulta en aumento de peso, cambios en el apetito sexual y caída del cabello. El desconocimiento sobre los efectos secundarios derivados del uso continuado de anticonceptivos hormonales orales ha afectado su efectividad, es fundamental que se advierta a estas mujeres sobre el uso de este método anticonceptivo, la forma más correcta de tomarlos, que pueden o no pueden utilizarlos y sobre todo, informar correctamente sobre los riesgos y beneficios que pueden tener los anticonceptivos orales. Este estudio tuvo como objetivo demostrar e informar el conocimiento de los académicos de la Facultad de Guaraí - TO, sobre las consecuencias del uso prolongado y continuo de anticonceptivos orales, que muchas veces se utilizan sin prescripción médica. El papel de los profesionales de la salud es fundamental a la hora de 
orientar e indicar el anticonceptivo ideal para cada mujer, con el fin de evitar una anticoncepción errónea y generar así efectos y problemas diferentes en la vida de cada mujer.

Palabras clave: Conocimiento; Anticonceptivo oral; Uso extendido; Consecuencias.

\section{Introdução}

Mudanças são constantes, e ao longo dos anos a sociedade vem se modificando culturalmente. Com isso, se têm uma melhor percepção sobre relação sexual, atitudes e pensamentos. Essa mudança vem influenciado no comportamento de vários jovens que, precocemente, inicia sua vida sexual mais cedo (Almeida \& Assis, 2017). Ter uma vida sexual ativa traz muitas preocupações, principalmente para as mulheres, sobretudo a gravidez indesejada, com isso, a utilização de métodos contraceptivos passa a ser uma ferramenta grandiosa e muito importante quando o assunto é planejamento familiar, ressaltando ainda a prevenção de Infecções Sexualmente Transmissíveis (IST’s) (Gonçalves \& De Moura Gomes, 2019).

São denominados métodos contraceptivos hormonais aqueles que possuem em sua presença hormônios, como o estrogênio e a progesterona que podem ser de forma associada ou isolada. Esses hormônios têm ação de inibir o amadurecimento dos óvulos, e com isso não ocorre a ovulação. Os contraceptivos hormonais orais são os métodos mais utilizados entre mulheres. A utilização se dá tanto para prevenção de uma gravidez, ou até mesmo para normalização do ciclo menstrual. Existem também outras alternativas de contraceptivos, tal como, os métodos cirúrgicos conhecidos de laqueadura e vasectomia considerados contraceptivos definitivos e os dispositivos intrauterino sem hormônio (de Freitas, Ceron \& Nowacki, 2019).

Entre os métodos citados acima, a contracepção hormonal é um dos mais utilizado pelas mulheres do Brasil e do mundo. No Brasil, são estimados $23 \%$ das mulheres com idade considerada reprodutiva usuárias dos anticoncepcionais hormonais (Araujo, et al., 2016). Para Herter e Acceta (2001), o sucesso desse medicamento está voltado para à sua eficácia (cerca de 99,9\%, se tomado corretamente), outro motivo, é por não interferir na vida sexual e pela segurança.

Marmitt, (2006), ressalta que, a utilização desse método contraceptivos proporciona inúmeros benefícios, como redução das cólicas, diminuição da acne, diminuição de casos de anemia, regularização do ciclo menstrual, proteção contra o câncer de ovário, câncer de mama e gravidez. Já Bouzas et al., (2004) afirma que, após a primeira ingestão, são comuns relatos de diversos sintomas. Os efeitos colaterais mais frequentes, associados a esses medicamentos são: dores de cabeça, náuseas, vômitos, tonturas, irritabilidade, aumento do apetite com consequência ganho de peso, alterações no apetite sexual e queda considerável de cabelo. Spanhol (2008), relatou que:

"O uso de forma contínua tem como consequência efeitos colaterais que variam desde alterações leves e reversíveis como hiperpigmentação e aumento de peso, até manifestações clínicas graves, como os eventos tromboembólicos. Os eventos tromboembólicos ocorrem dentro do primeiro ano de uso de anticoncepcional hormonal, principalmente após o quarto mês do início do uso. Após um ano o tempo de uso dos contraceptivos orais não altera o risco para trombose venosa, não havendo dessa forma, risco acumulado com o passar dos anos. Assim, trocas desnecessárias dos contraceptivos hormonais podem elevar o risco para uma trombose venosa em cada troca."

O risco a essa doença está relacionado diretamente à dose do estrogênico e o etinilestradiol, que quando presente na corrente sanguínea, tem a formação da trombina aumentada, elevação da coagulação e diminuição des inibidores, gerando efeito pró-coagulante leve (Brito, Nobre \& Vieira, 2010).

“O conhecimento sobre métodos contraceptivos pode contribuir para que os indivíduos escolham o que é mais adequado ao seu comportamento sexual e condições de saúde, bem como para seu uso de forma correta. Assim, esse conhecimento deve estar relacionado à prevenção da gravidez indesejada, do aborto provocado, da mortalidade materna e de outros agravos à saúde relacionados à morbimortalidade reprodutiva (Penaforte, et al., 2010).” 
De acordo com os autores Da Silva, Pinto e De Lima (2020) mulheres hipertensas e com doenças cardiovasculares usuárias dos métodos orais, tem apresentado maior risco a trombose arterial e a acidente vascular hemorrágico (AVC). Deste modo, é essencial que essas mulheres sejam alertadas sobre o uso desse método contraceptivo, a forma mais correta de tomálos, quem pode ou não fazer uso e especialmente, informar corretamente dos benefícios e riscos que esses contraceptivos podem ocasionar.

A falta de conhecimento sobre os efeitos colaterais oriundos do uso contínuo dos contraceptivos hormonais orais tem afetado sua eficácia. Leite (2003) ressalta que, mulheres que fazem uso do método oral, com a intenção de prevenção, cerca de 40\% delas interrompem o uso nos 12 primeiros meses, resultado como esse se dá pela ausência de um profissional de saúde especializado, já que muitas das pacientes fazem uso do medicamento sem uma prescrição médica.

Devido à ausência de estudos sobre o tema em questão, este trabalho teve como objetivo, evidenciar e relatar o conhecimento de acadêmicas da Faculdade de Guaraí - TO, sobre as consequências do uso prolongado e contínuo de anticoncepcional oral, levando em consideração o grande consumo deste medicamento, que por muitas vezes é utilizado sem indicação e prescrição de um profissional de saúde, o que pode acarretar para o surgimento de diversos problemas de saúde.

\section{Metodologia}

Esta pesquisa é de cunho quantitativo-qualitativo baseou-se em coleta de dados e por demonstração estatística dos resultados obtidos.

\subsection{Caracterização da área de estudo}

A presente pesquisa foi desenvolvida na Faculdade Guaraí (IESC-FAG), instituição situadas no município de Guaraí TO, Brasil, que se encontra sob as coordenadas $08^{\circ} 50^{\prime} 03^{\prime \prime}$ S e $48^{\circ} 30^{\prime} 37^{\prime}$ W W de longitude e altitude de $259 \mathrm{~m}$, a aproximadamente $184 \mathrm{~km}$ da Capital do Estado, Palmas, conforme consta na figura 1. O Município possui cerca de 26.165 habitantes (IBGE, 2020). A densidade demográfica equivale a 10,23 hab./km² (IBGE, 2010).

Figura 1. Mapa do estado do Tocantins, dando destaque ao município de Guaraí (TO).

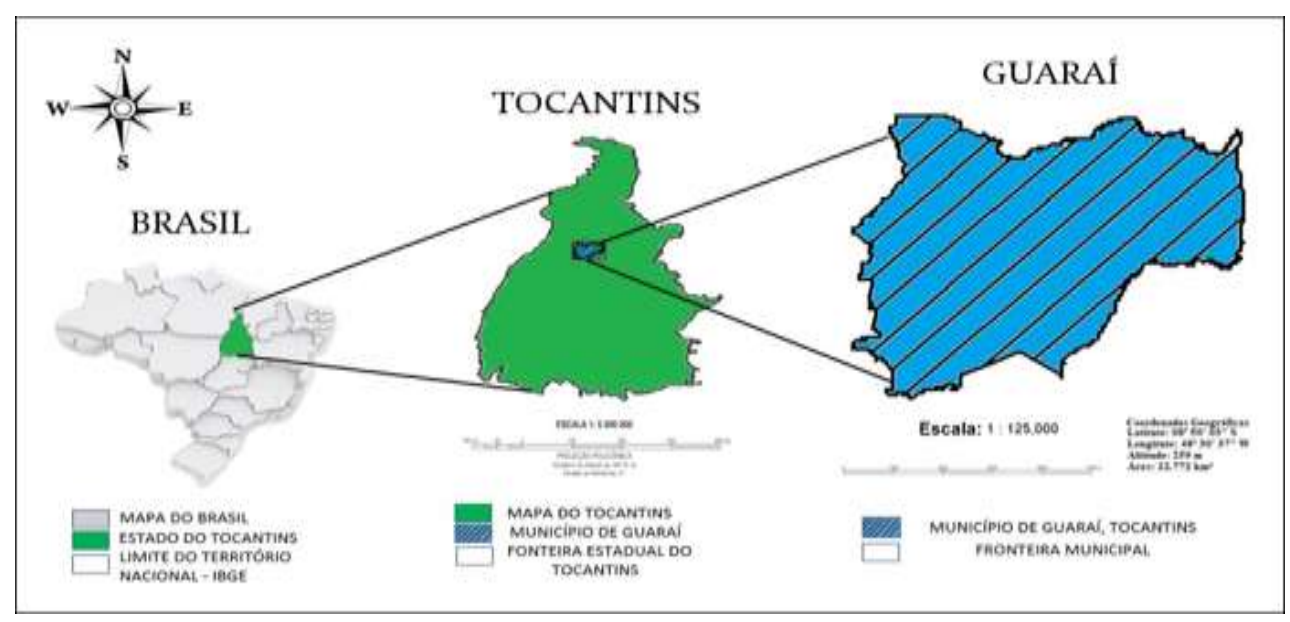

Fonte: da Silva, et al., (2018).

\subsection{Coleta de dado}

A coleta de dados foi inspirada no método de Marconi et al. (2008), em que se obteve os dados qualitativos e quantitativos através da aplicação de um questionário, e os dados serão obtidos por meio da plataforma digital Google Forms. A entrevista foi $100 \%$ online devido a pandemia do novo coronavírus (Covid-19). 
O questionário utilizado continha duas (2) perguntas subjetivas e onze (11) perguntas fechadas de múltipla escolha, que segundo Freitas, Barros e Carvalho (2019), favorece uma melhor interpretação das perguntas e ajuda na análise de dados. A presente pesquisa tinha como número alvo cem (100) mulheres entre 18 e 39 anos aptas a responder. Todas as perguntas eram de autoria própria, porém baseadas em artigos já publicado com relação da mesma temática. A análise dos dados consistirá na apresentação estatística descritiva das informações obtidas.

Foram estabelecidas treze perguntas semiestruturadas contidas no questionário empregue para mulheres entre 18 e 39 anos da Faculdade Guaraí em relação as consequências do uso contínuo do anticoncepcional oral, para análise do conhecimento das mesmas, as perguntas estão contidas no Quadro 1 a seguir.

Quadro 1. Questões utilizadas no questionário aplicado as mulheres acerca do anticoncepcional oral.

\begin{tabular}{|l|}
\hline Quantos anos você tem? \\
\hline Qual a sua escolaridade? \\
\hline Você sabe o que é um método contraceptivo? \\
\hline Você já ouviu falar método contraceptivo oral? \\
\hline Dos exemplos abaixo, quais métodos de contracepção você já ouviu falar: \\
\hline Sobre a pílula anticoncepcional, você faz ou já fez uso desse método contraceptivo? \\
\hline Qual método contraceptivo você usa? \\
\hline Se você usa o método oral (pílula anticoncepcional), qual foi a indicação desse medicamento? \\
\hline Quem fez a indicação do seu anticoncepcional? \\
\hline Dos riscos citados abaixo, quais você acredita que são efeitos do uso prolongado? \\
\hline Com o uso decorrente desse medicamento você sentiu alguma mudança em seu corpo? \\
\hline De acordo com o seu conhecimento, qual a maior consequência do uso contínuo do anticoncepcional oral? \\
\hline $\begin{array}{l}\text { Conhecendo todos os riscos que esse medicamento pode trazer, você indicaria o mesmo para outras mulheres que pretende começar a } \\
\text { usar? }\end{array}$ \\
\hline
\end{tabular}

Fonte: Autores.

\subsection{Análise de dados}

O critério estatístico utilizado foi descritivo com análise qualitativa e quantitativa (Michel, 2005). A pesquisa foi realizada com cem (100) mulheres entre 18 e 39 anos, acadêmicas da Faculdade Guaraí, que se encontravam na faixa etária classificada como idade reprodutiva (10-49 anos), porém participaram somente mulheres acima de 18 anos, por estas poderem assinar o Termo de Consentimento Livre e Esclarecido (TCLE). A escolha de mulheres nessa faixa etária, foi feita por estas possuírem grande números de consumos do medicamento em questão.

\section{Resultados e Discussão}

Foram avaliadas respostas de 100 (cem) mulheres, as mesmas são egressas da Faculdade Guaraí. A presente pesquisa, demonstra que 55\% ( $\mathrm{n}=55)$ das mulheres pesquisadas possuíam idade entre 18 a 24 anos, seguindo de $24 \%$ ( $\mathrm{n}=24$ ) com idade de 25 a 32 anos, e por fim de 33 a 39 anos com percentual de 21\% (n=21). Dentre essas, 69\% (n=69) das pesquisadas possuíam o ensino superior incompleto e $31 \%(\mathrm{n}=31)$ das mesma, confirmam ter o ensino médio completo, considerando assim um número de 100 mulheres com o $3^{\circ}$ grau de escolaridade incompleto, esses resultados estão expostos na Tabela 1. 
Tabela 1. Dados referentes a idade e escolaridade das mulheres egressas da Faculdade Guaraí.

\begin{tabular}{lcc}
\hline \multicolumn{1}{c}{ Variável } & Total de participantes & $\%$ \\
\hline Faixa etária (anos) & 55 & $55 \%$ \\
$18-24$ & 24 & $24 \%$ \\
$25-32$ & 21 & $21 \%$ \\
$33-39$ & & $31 \%$ \\
\hline Escolaridade & 31 & $69 \%$ \\
\hline Ensino médio completo & 69 & \\
\hline Ensino superior incompleto & & \\
\hline
\end{tabular}

Fonte: Autores (2021).

\subsection{Conhecimento sobre métodos contraceptivos com especificidade no método oral}

Rodrigues (2015), afirma que conhecer é o primeiro passo para a construção de uma opinião crítica e reflexiva. De acordo com Gonçalves \& De Moura (2019), a falta de conhecimento das mulheres em relação ao método contraceptivo oral e o uso contínuo prolongado pode trazer vários efeitos adversos, entre os mais comuns estão as náuseas, dores de cabeça e ganho de peso. Para os autores, as mesmas estão correndo grande risco a saúde por não adquirirem conhecimento necessário sobre o medicamento.

A presente pesquisa abordou duas (2) questões que visam o conhecimento das acadêmicas sobre esses métodos. As perguntas estão tituladas em: Você sabe o que é um método contraceptivo? Pergunta essa que, teve como resultado "Sim" um percentual de $98 \%(n=98)$ e "Não" com $2 \%(n=2)$ dando a entender que essas acadêmicas possuem um conhecimento necessário sobre o que é um método contraceptivo.

Outra pergunta que foi desenvolvida está descrita: Você já ouviu falar em método contraceptivo oral? A mesma teve como resultado "Sim" o percentual de 97\% $(n=97)$ e "Não" com 3\% $(n=3)$ deixando evidente que, em algum momento da vida de mais da maioria das académicas as mesmas já terem presenciado a pronuncia ou o conceito desse método.

Para Vieira et al. (2020) ter conhecimento sobre métodos contraceptivos é um fator primordial, pois ter noção sobre esses métodos contribui para que indivíduos escolham pôr si só o mais adequado ao seu comportamento sexual, levando em conta suas condições de saúde. Desse modo, esse conhecimento está relacionado à prevenção de diversos efeitos colaterais, como ganho de peso, náuseas, diminuição da libido, além de evitar outros agravos a saúde como a gravidez indesejada, da mortalidade materna e do aborto.

No presente estudo, foi discutido o conhecimento das acadêmica acerca dos métodos de contracepção, o resultado da pesquisa está exposto na Tabela 2 a seguir.

Tabela 2. Conhecimento de acadêmicas acerca dos métodos de contracepção.

\begin{tabular}{lcc}
\hline \multicolumn{1}{c}{ Tipos de Contracepção } & Quantidade (n) & (\%) \\
\hline Dispositivo intrauterino (DIU) & 92 & $92 \%$ \\
Laqueadura ou vasectomia & 87 & $87 \%$ \\
Anticoncepcional injetável & 91 & $91 \%$ \\
Camisinha masculina e feminina & 93 & $93 \%$ \\
Pílula anticoncepcional & 99 & $99 \%$ \\
Adesivo & 3 & $3 \%$ \\
Tabelinha & 1 & $1 \%$ \\
Diafragma & 1 & $1 \%$ \\
\hline
\end{tabular}

Fonte: Autores (2021). 
Dados expostos na Tabela 2, deixam evidenciado que as entrevistadas possuem um bom conhecimento sobre os diferentes tipos de contracepção, sendo método oral (pílula anticoncepcional) a mais conhecida e usada por elas com percentual de 99\% (n=99). Logo atrás, vem a camisinha masculina e feminina, conhecido também como condom, com 93\% das acadêmicas, equivalente ao número de 93 acadêmicas. Resultado semelhante encontrado na pesquisa dos autores Belo \& Silva (2004), onde foi revelado que 94,2 e 91,7\% das entrevistadas obtêm um maior conhecimento sobre o anticoncepcional oral e o condom, e as mesmas veem esses métodos como os mais frequentes.

Uma parcela considerável das acadêmicas relatou conhecer outros tipos de contracepção, entre eles, o mais citado por elas foi o dispositivo intrauterino (DIU), com um percentual de 92\% ( $\mathrm{n}=92$ ). Dispositivo intrauterino (DIU), é um dos métodos contraceptivos mais utilizados no mundo, principalmente em países emergentes. O número de usuárias desse método passa de 150 milhões (Freitas et al., 2011).

Outro meio de contracepção com alto índice de conhecimento relatado pelas acadêmicas com o percentual de $87 \%$ (n= 87) é o método contracepitivo definitivo, conhecido como laqueadura e vasectomia. Métodos como esse são utilizados com intuito de neutralizar a possibilidade de gravidez, além de evitar o consumo de alguns métodos contraceptivos com presença de hormônios sintéticos em sua fórmula (estrogênio e o progestogênio), evitando o surgimento de diversos efeitos colaterais oriundos do consumo prolongado desses medicamentos.

Outros métodos foram citados pelas acadêmicas, e teve como resultado o anticoncepcional injetável com número de 91\% ( $\mathrm{n}=91$ ) como um dos mais conhecidos entre as usuárias. O Anticoncepcional Injetável é considerado o método com mais eficácia dentre os anticoncepcionais reversíveis, com um considerável número de usuárias no Brasil e em outros países do mundo. No Brasil, os anticoncepcionais injetáveis podem ser ofertados pelo Sistema Único de Saúde (SUS). Os mesmos, podem ser classificados por duas apresentações, sendo eles combinados (mensais) e os exclusivos de progestágeno (trimestrais) (Da Silva Farias et al., 2018).

\subsection{Importância da indicação e utilização do método ideal de contracepção}

Souza (2017), afirma que na escolha do método contraceptivo ideal, tem que ser levado em consideração aquele que atende todas às necessidades individuais e do casal. A escolha do método contraceptivo tende a ser feito mediante a um acompanhamento, pois o uso de qualquer tipo de contracepção sem um acompanhamento médico, pode aumentar os riscos de desenvolver diversos tipos de doenças (Azevedo, Campos \& Vidal, 2020).

O uso do anticoncepcional oral tem diversos benefícios, como regulação do ciclo menstrual, diminuição das cólicas menstruais e TPM, além de ter 99,9\% de eficácia se tomado corretamente, como já mencionado na pesquisa. Mas, quando o mesmo é utilizado por um período prolongado, pode surgir diversos problemas provocando o agravamento e assim aumentado a coagulação sanguínea podendo contribuir com o surgimento de trombose, maiores probabilidades de desenvolver câncer, infertilidade, além de seus efeitos mais comuns como dores de cabeça e náuseas (Gonçalves \& De Moura Gomes, 2019).

$\mathrm{Na}$ presente pesquisa foi perguntado sobre a utilização dá pílula anticoncepcional, se as acadêmicas fazem ou já fizeram uso desse método contraceptivo oral. O resultado da pesquisa foi que $50 \%$ ( $n=50$ ) fazem uso do método contraceptivo mencionado, seguido por $46 \%(n=46)$ dizem já terem tomado o anticoncepcional oral, mas hoje não fazem mais uso e apenas $4 \%(n=4)$ relataram nunca terem feito uso desse método. Resultados esses semelhantes encontrados na pesquisa dos autores Siqueira, Sato e Santiago (2017), onde o número de questionários alcançados foram 205 participantes. A faixa etária abordada foi de 18 a 51 anos, sendo as idades 23 e 25 as mais predominantes. A pesquisa dos mesmos autores apontou que de duzentos e cincos mulheres (205) 86,4\% faziam uso do anticoncepcional oral e 14,1\% não utilizava.

A seguir, o resultado envolvendo duas (2) perguntas presente no questionário, ambas questões acerca da indicação e utilização do método contraceptivo, o resultado está exposto na Tabela 3. 
Tabela 3. Dados referente ao tipo de contracepção e quem fez a indicação desse método.

\begin{tabular}{lcc}
\hline Qual método contraceptivo você usa? & quantidade (n) & $\mathbf{( \% )}$ \\
\hline Camisinha & 18 & $18 \%$ \\
Pílula anticoncepcional & 37 & $37 \%$ \\
Anticoncepcional injetável & 10 & $10 \%$ \\
Nenhum & 20 & $20 \%$ \\
Implante anticoncepcional & 6 & $6 \%$ \\
Dispositivo intrauterino (DIU) & 5 & $5 \%$ \\
Anel vaginal & 3 & $3 \%$ \\
Laqueadura & 1 & $1 \%$ \\
\hline Quem fez a indicação do seu anticoncepcional? & & \\
\hline Médico & 74 & $74 \%$ \\
Farmacêutico & 11 & $11 \%$ \\
Amiga & 10 & $10 \%$ \\
Internet & 2 & $2 \%$ \\
Namorado & 3 & $3 \%$ \\
\end{tabular}

Fonte: Autores (2021).

Ter conhecimento sobre os diferentes tipos de contracepção é fundamental na hora de escolher o método ideal para seu corpo, mas a escolha deve ser feita mediante um médico, pois, segundo a Organização Mundial da Saúde (OMS), a utilização de qualquer método contraceptivo é proveniente de contraindicação, segundo os critérios de elegibilidade estabelecidos.

Diante do exposto na Tabela 3, fica evidenciado que o anticoncepcional oral com percentual de 37\% (n=37) é o mais utilizado pelas acadêmicas, e isso se dá pela alta porcentagem de eficácia, vale ressaltar que o número para o sucesso desse medicamento depende da ingestão diária, que pode levar a uma taxa de falha de $0,01 \%$ para aproximadamente $8 \%$ quando se tem uma má administração (Rios, et al., 2021). Logo em seguida, vem o método contraceptivo de barreira, conhecido popularmente por camisinha, com um número de 18 acadêmicas ( $n=18)$, esse, está em alta pois tem seu custo-benefício baixo e de fácil acesso.

Outros meios de contracepção foram relatados e exposto na tabela. Com percentual de 10\% ( $\mathrm{n}=10)$ o anticoncepcional injetável foi um dos mais citados, isso porque, ele é de fácil acesso, sendo ofertado pelo Sistema Único de Saúde (SUS), os mesmos podem ser administrados trimestralmente e mensalmente. Tsehaye et al., (2011), aponta que o aumento da satisfação das usuárias dos anticoncepcionais injetáveis tem relação com a longa duração (dispensando assim o controle diário, como os anticoncepcionais orais), seu fácil acesso e sua grande eficácia.

Falando de suas desvantagens, Freitas e Giotto, (2018) aponta que diferentemente do anticoncepcional oral o método injetável não traz uma regulação do ciclo menstrual e sim uma irregularidade. Os mesmos autores apontam como desvantagem também, aumento de peso e o grande risco de infertilidade.

Resultados como esses foram encontrados no trabalho de Lago et al., (2020), onde teve o método de contracepção oral (pílula) com porcentagem de $27 \%$ sendo assim o mais utilizado, logo atrás vem o preservativo masculino (19\%) seguido pelo anticoncepcional injetável com percentual de 10,4\%.

Quando relacionado a indicação do método contraceptivo oral, 74\% $(n=74)$ e 11\% $(n=11)$ das acadêmicas relataram ter procurado um profissional da saúde (médico ou enfermeiro) e este ter indicado o método contraceptivo oral para ela. Mas, um número considerado de 10 (10\%) acadêmicas relataram ter usado o medicamento por indicação de amiga ou terceiros, como internet (2\%) e namorado (3\%).

Alves e Lopes, (2007) ressalta que a indicação de qualquer método contraceptivo deve ser feita por um profissional de 
saúde, pois esse profissional sempre levará em consideração as características de cada usuárias, pois, alguns métodos podem originar diversos efeitos adversos.

A seguir, resultado da pesquisa sobre qual a indicação do anticoncepcional oral. No final dessa etapa da pesquisa, obteve-se um número de 86 acadêmicas aptas a responderem. Para fins de exclusão, era considerado apenas mulheres usuárias de anticoncepcionais orais. No total foram excluídas 17 respostas, por essas não entrarem no critério de avaliação. E esses resultados estão demostrado na Tabela 4.

Tabela 4. Resultados sobre qual indicação do anticoncepcional oral.

\begin{tabular}{lcc}
\hline \multicolumn{1}{c}{ Tipos de indicação } & Quantidade (n) & $(\mathbf{\% )}$ \\
\hline Evitar gravidez & 67 & $77,9 \%$ \\
Controle hormonal & 17 & $19,8 \%$ \\
Regulação do ciclo menstrual & 26 & $30,2 \%$ \\
Diminuição das cólicas menstruais & 16 & $18,6 \%$ \\
Combater micropolicistos & 2 & $2,4 \%$ \\
Tratamento de miomas uterinos. & 1 & $1,2 \%$ \\
Redução do fluxo menstrual por motivos de anemia & 1 & $1,2 \%$ \\
\hline
\end{tabular}

Fonte: Autores (2021).

A utilização do método contraceptivo oral, se dá após a indicação de um médico. Diante da necessidade exposta pela paciente, o médico indica o melhor anticoncepcional para suprir a sua necessidade. Diante disso, obteve-se 7 diferentes tipos de indicação do anticoncepcional oral. A indicação para evitar uma gravidez indesejada foi a que obteve maior percentual, com um número de 77,9\% (n=67) acadêmicas afirmando utilizar o método de contracepção oral, a fim de evitar uma gravidez.

Outros problemas foram citados pelas acadêmicas, inclusive a utilização do anticoncepcional oral para a regulação do ciclo menstrual, que teve como resultado o percentual de 19,8\% ( $\mathrm{n}=17)$ afirmando que faz uso do mesmo para regulação do seu ciclo. Almeida e Assis (2017), afirmam que a adesão de contraceptivos hormonais, com exclusividade hormonal oral, tem como motivo os benefícios que esses medicamentos proporcionam, como a diminuição de cólicas, regulação do ciclo menstrual, gravidez ectópica, proteção contra câncer do ovário, além de participar no controle hormonal.

Obteve-se também a indicação do método oral para a diminuição de cólicas menstruais, com o resultado de 18,6\% $(\mathrm{n}=16)$. Cólicas menstruais é um transtorno ginecológico, na língua cientifica chamado também de dismenorreia ou síndrome de dor menstrual. O medicamento oral é importante e indicado pois o mesmo, ajuda a diminuir as intensas contrações das musculaturas uterinas.

Obteve-se também outros 3 tipos de indicação por meio do contraceptivo oral, esses, com pouca porcentagem, mostrando assim a baixa ocorrência desses casos. As indicações são, para combater micropolicistos, que teve como percentual o número de 2,4\% ( $\mathrm{n}=2)$ das acadêmicas. Tratamento de miomas uterinos que correspondeu a 1,2\% $(\mathrm{n}=1)$ das acadêmicas, e redução do fluxo menstrual por motivos de anemia que obteve o número de $1,2 \%$, correspondente a 1 acadêmica.

A indicação do anticoncepcional se dá de acordo com a necessidade de cada usuária, por isso se torna necessário a indicação de um profissional de saúde.

\subsection{Anticoncepção oral e seus efeitos adversos}

Diante do resultado obtido, e do uso intenso de contraceptivos hormonal oral, estudos tem crescidos e evidenciado cada vez mais que a utilização desse medicamento pode originar diversos malefícios a saúde da mulher (Dos Santos, 2020).

A presente pesquisa evidenciou que, a utilização desse medicamento oral é responsável por diversos problemas relacionado a saúde. Isso se comprova a seguir, onde as acadêmicas relataram por meio da pesquisa, efeitos adversos oriundos 
da utilização do anticoncepcional oral. O resultado está exposto na Tabela 5.

Tabela 5. Efeitos oriundos da anticoncepção oral.

\begin{tabular}{lcc}
\hline \multicolumn{1}{c}{ Efeitos adversos } & Quantidade (n) & (\%) \\
\hline Ganho de peso & 50 & $50 \%$ \\
Alteração no humor & 47 & $47 \%$ \\
Ansiedade & 26 & $26 \%$ \\
Queda de cabelo & 22 & $22 \%$ \\
Retenção de líquidos & 8 & $8 \%$ \\
Diminuição da libido & 7 & $7 \%$ \\
Espinha & 4 & $4 \%$ \\
Nenhum & 16 & $16 \%$
\end{tabular}

Fonte: Autores (2021).

Efeitos colaterais oriundos da anticoncepção oral, são bastante recorrentes nas suas usuárias, e isso se torna mais frequente quando, as mesmas, fazem uso prolongado desse medicamento. A presente pesquisa obteve um número de 7 reações adversas, resultados esses apresentados na Tabela 5 .

As usuárias, por meio da pesquisa deixaram notório que, ganho de peso com o percentual de $50 \%(\mathrm{n}=50)$ é o efeito mais comum quando se faz uso desse medicamento, seguida pela alteração de humor com percentual de $47 \%$ ( $\mathrm{n}=47$ ). Os resultados obtidos são semelhantes a diversas pesquisas. Na pesquisa de, Da Silva Carrias et al., (2019) titulada em "Efeitos adversos associados a uso de contraceptivos orais em discentes", os autores relataram que $63,8 \%$ das entrevistadas já sentiram algum sintoma adverso relacionado ao uso do método contraceptivo oral, sendo os mais constantes aumentos de peso com o percentual de $32,4 \%$, seguida pela alteração de humor, com $24,3 \%$.

Outros efeitos adversos foram relatados pelas acadêmicas, ansiedade foi uma das mais relatadas por elas e apresentam um percentual de $26 \%(\mathrm{n}=26), \log$ atras vem a queda de cabelo com $22 \%$ das acadêmicas $(\mathrm{n}=22)$. Sousa et al. (2017), afirma em sua pesquisa que, as usuárias do método de contracepção hormonal oral relataram sentir efeitos colaterais, como a mudança de humor e aumento da ansiedade. Bouzas et al. (2004) afirma em seu trabalho que, os efeitos mais comumente oriundos da anticoncepção hormonal oral são, aumento do apetite levando assim a um ganho de peso, queda de cabelo e diminuição da libido.

Além disso, outras 3 reações foram citadas por elas, sendo a retenção de líquido com percentual de 8\% (n=8), diminuição da libido com $4 \%(\mathrm{n}=4)$ e por fim, com um número de 4 acadêmicas, correspondente a $4 \%$ relataram o surgimento de espinhas. $\mathrm{O}$ uso do método contraceptivo oral pode causar efeitos adversos, como a queda da libido, aparecimento espinhas e cravos, além do aumento de peso que é resultante do ganho de excessivo apetite (Costa et al., 2017). Resultados como esses, foram encontrados na pesquisa dos autores Pretes e Quadros (2021), onde, diminuição da libido com 34,3\% e retenção de liquido (inchaço) 26,3 foram um dos maiores efeitos sentidos por essas mulheres.

\section{Conclusão}

Baseado nos dados encontrados na pesquisa, foi observado que as acadêmicas possuem conhecimento relevante acerca das consequências decorrente ao uso prolongado do anticoncepcional oral. As mesmas, deixam evidente ter consciência da sua escolha de contracepção, mesmo sabendo dos riscos oferecidos por esse medicamento. A escolha desse método de contracepção se dá por ele possuir alta taxa de eficácia, chegando até 99,9 se tomado corretamente, segundo os autores e por ter seu custo benéfico baixo.

O fato que contribui para a elevação do número de usuárias desse medicamento está associado à sua indicação. Notou- 
se que, a utilização do anticoncepcional oral além de ser indicado no planejamento familiar, evitando a gravidez indesejada, ele tem sido utilizado para auxiliar no melhoramento de diversas partes do corpo, sendo indicada na hora de regulação do ciclo menstrual, redução das cólicas menstruais, proteção contra o câncer de ovário, diminuição de casos de anemia e proteção contra o câncer de mama.

A pesquisa evidenciou que a utilização a longo prazo pode ser crucial no surgimento dos efeitos colaterais. Os feitos adversos mais relatados por elas foram o ganho de peso com 50\%, alteração do humor $47 \%$, seguido por ansiedade com $26 \%$. A presente pesquisa relatou que o profissional de saúde é procurado por $75 \%$ das participantes, sendo que o restante $15 \%$ das participantes acabam procurando uma orientação de terceiros, sendo amigo, internet, e o companheiro sexual (namorado).

Diante do exposto, espera-se que a presente pesquisa contribua para uma melhor compreensão dos riscos e benefícios que esse medicamento pode trazer. Para estudos futuros, fica a necessidade de aplicação de ações afim de conscientizar as usuárias sobre o risco oferecidos por esse medicamento. Pensando em bem-estar, fica notório que a utilização sem prescrição médica é um fator a ser explorado com mais clareza, pois, é imprescindível a atuação do profissional de saúde na hora de indicar e contraindicar o anticoncepcional ideal a cada mulher, afim de evitar a contracepção de forma errônea e gerando assim diversos efeitos e problemas a vida de cada uma.

\section{Referências}

Almeida, A. P. F. D., \& Assis, M. M. D. (2017). Efeitos colaterais e alterações fisiológicas relacionadas ao uso contínuo de anticoncepcionais hormonais orais. Rev Eletronica Atualiza Saude, 5(5), 85-93.

Alves, A. S., \& Lopes, M. H. B. D. M. (2007). Locus de Controle e escolha do método anticoncepcional. Revista brasileira de Enfermagem, 60, $273-278$.

Araujo, A. B. R., Parreira, A. M., Valadares, C. A., Tourinho, C. A., Pinto, P. V., \& Souza, J. H. K. (2016). Anticoncepcionais hormonais contendo apenas progestágenos e seus principais efeitos. Artigo Científico (Curso de Medicina)-Faculdade de Minas, FAMINAS-BH, Belo Horizonte-MG.

Belo, M. A. V., \& Silva, J. L. P. (2004). Conhecimento, atitude e prática sobre métodos anticoncepcionais entre adolescentes gestantes. Revista de Saúde Pública, 38, 479-487.

Bouzas, I., Pacheco, A., \& Eisenstein, E. (2004). Orientação dos principais contraceptivos durante a adolescência. Adolescência e Saúde, 1(2), 27-33.

Brito, M. B., Nobre, F., \& Vieira, C. S. (2011). Contracepción hormonal y sistema cardiovascular. Arquivos brasileiros de Cardiologia, 96, e81-e89.

Costa, B. A. (2017). Risco de trombose associado à terapia dos anticoncepcionais hormonais: uma revisão de literatura .

da Silva, B. B., Rocha, L. G., de Oliveira Silveira, L. P., de Carvalho, A. V., \& Guimarães, A. P. M. (2018). Análise da qualidade da água do Ribeirão Tranqueira. Revista Ibero-Americana de Ciências Ambientais, 9(8), 11-25. DOI: https://doi.org/10.6008/CBPC2179-6858.2018.008.0002.

da Silva Carrias, D. T., Araújo, N. C., Meirelles, L. M. A., \& Neto, B. M. (2019). Efeitos adversos associados a uso de contraceptivos orais em discentes. Revista da Sociedade Brasileira de Clínica Médica, 17(3), 142-146.

da Silva, V. N. B., Pinto, H. V. D. C., \& De Lima, L. R. (2020). Efeitos colaterais e reações adversas relacionados ao uso de anticoncepcionais hormonais orais. Encontro de Extensão, Docência e Iniciação Científica (EEDIC), 7.

de Freitas, E. M., Ceron, R., \& Nowacki, L. (2020). Uso de anticoncepcionais orais e dispositivo intrauterino hormonal (Mirena ${ }^{\circledR)}$ relacionado ao risco de trombose venosa profunda (tvp). revista eletrônica biociências, biotecnologia e saúde, 13(26), 37-44.

dos Santos, R. L., de Oliveira Barbosa, A. D. L., Santana, A. L., Farias, J. V. C., de Macêdo, P. R., \& Farias, I. C. C. (2020). Os riscos do uso prolongado de contraceptivos hormonais. Research, Society and Development, 9(11), e69791110394-e69791110394. https://doi.org/10.33448/rsd-v9i11.10394.

Farias, A. G. D. S., Lima, A. C. S., Brasil, R. F. G., Moura, E. R. F., Cunha, M. D. C. D. S. O., \& Melo, F. M. D. S. (2018). Uso seguro de anticoncepcionais hormonais injetáveis segundo critérios médicos de elegibilidade. Rev. Pesqui. 368-373. https://doi.org/10.9789/2175-5361.2018.v10i2.368-373.

Fonseca, A. C. N., Gomes, A. T., \& Barreto, J. G. (2015). Distribuição de anticoncepcionais em uma farmácia básica no município de São José do CalçadoES. Acta Biomedica Brasiliensia, 6(1), 10-20.

Freitas, F. S., \& Giotto, A. C. (2018). Conhecimento sobre as consequências do uso de anticoncepcional hormonal. Revista de Iniciação Científica e Extensão, $1(2), 91-95$.

Freitas, F., Menke, C., \& Rivoire, W. (2011). Rotinas em Ginecologia. ed. Artmed Editora.

Freitas, K. T. S., Barros, J. B., \& de Carvalho, A. V. (2019). Controle e prevenção de pulgas em cães e gatos: análise das ações dos tutores. Agriculturae, 1(1), 13-20. https://doi.org/10.6008/CBPC2674-645X.2019.001.0002. 
Research, Society and Development, v. 10, n. 15, e349101522949, 2021

(CC BY 4.0) | ISSN 2525-3409 | DOI: http://dx.doi.org/10.33448/rsd-v10i15.22949

Gonçalves, B. S., \& de Moura Gomes, G. (2019). Consequências decorrentes do uso prolongado de Contraceptivos Medicamentosos: Uma Revisão Bibliográfica/Consequences arising from prolonged use of Medication Contraceptives: A Bibliographic Review. ID on line Revista De Psicologia, 13(45), 90101.

Herter, L. D., \& SG, A. (2001). Anticoncepção e gestação na Adolescência. J Pediatr (Rio J), 77, 170-8.

Lago, T. D. G. D., Kalckmann, S., Alves, M. C. G. P., Escuder, M. M. L., Koyama, M., \& Barbosa, R. M. (2020). Diferenciais da prática contraceptiva no Município de São Paulo, Brasil: resultados do inquérito populacional Ouvindo Mulheres. Cadernos de Saúde Pública, 36. DOI: https://doi.org/10.1590/0102311 X00096919.

Leite, I. D. C. (2003). Descontinuação de métodos anticoncepcionais no Nordeste do Brasil, 1986-1991. Cadernos de Saúde Pública, 19, $1005-1016$.

Martins, L. B. M., Costa-Paiva, L., Osis, M. J. D., Sousa, M. H. D., Pinto Neto, A. M., \& Tadini, V. (2006). Conhecimento sobre métodos anticoncepcionais por estudantes adolescentes. Revista de Saúde Pública, 40, 57-64.

Michel, M. H. (2005). Metodologia da pesquisa em ciências sociais: Um guia prático para acompanhamento da disciplina e elaboração de trabalhos monográficos. São Paulo: Atlas.

Penaforte, M. C. L. F., da Silva, L. R., dos Santos Esteves, A. P. V., da Silva, R. F., dos Santos, I. M. M., \& Silva, M. D. B. (2010). Conhecimento, uso e escolha dos métodos contraceptivos por um grupo de mulheres de uma unidade básica de saúde em Teresópolis, RJ. Cogitare Enfermagem, 15(1). DOI: http://dx.doi.org/10.5380/ce.v15i1.17183.

PRETES, N. S., \& QUADROS, P. G. B. D. (2021). Avaliação dos efeitos adversos produzidos pela utilização de contraceptivos hormonais.

Ranieri, C. M., \& Silva, R. F. (2011). Atenção farmacêutica no uso de métodos contraceptivos. Monografia (Especialização em Farmacologia) -Universitário Filadélfia de Londrina UNIFIL, Londrina-PR.

Rios, A. R., de Sena, A. D., Krug, B. R., de Oliveira Dantas, E. K., Ferronato, E. C. B., Bomfim, J. Q., \& Guimarães, R. M. G. C. (2021). Fatores relacionados à escolha de métodos contraceptivos na adolescência: uma revisão de literatura. Revista Eletrônica Acervo Saúde, 13(5), e6942-e6942. DOI: https://doi.org/10.25248/reas.e6942.2021.

Rodrigues, D. N. J. (2015). Avaliação do conhecimento da população sobre formas de transmissão e medidas preventivas da Toxoplasmose em Mossoró-RN.

Siqueira, T. C., Sato, M. D. O., \& Santiago, R. M. (2017). Reações adversas em usuárias de anticoncepcionais orais. Revista Eletrônica de Farmácia, 14(4). https://doi.org/10.5216/ref.v14i4.45511.

Souza, É. D. F. D. (2017). A importância do planejamento familiar com uso adequado dos métodos anticoncepcionais na Estratégia de Saúde da Família.

Souza, F. C. B. D. (2017). Ansiedade, depressão e qualidade de vida em mulheres usuárias de contraceptivos hormonais orais.

Spanhol, K. T., \& Panis, C. (2013). Contraceptivos orais e eventos trombóticos. Infarma-Ciências Farmacêuticas, 21(3/4), 7-13.

Tsehaye, W. T., Mengistu, D., Birhanu, E., \& Berhe, K. K. (2013). Assessment of preference and its determinant factors to ward modern contraceptive methods among women of reproductive age group in shire Indaselassie town, northern Ethiopia, 2011. International journal of family medicine, 2013. https://doi.org/10.1155/2013/317609.

World Health Organization, \& World Health Organization. Reproductive Health. (2010). Medical eligibility criteria for contraceptive use. World Health Organization. 